\title{
Measured Response of Local, Mid-range and Far-range Discontinuities of Large Metal Groundplanes using Time Domain Techniques
}

\author{
T. Schrader ${ }^{1}$, K. Münter ${ }^{1}$, S. Battermann ${ }^{2}$, and H. Garbe ${ }^{2}$ \\ ${ }^{1}$ Physikalisch-Technische Bundesanstalt, AG 2.21, Bundesallee 100, 38116 Braunschweig, Germany \\ ${ }^{2}$ Institut für Grundlagen der Elektrotechnik und Messtechnik, Universität Hannover, Appelstraße 9a, 30167 Hannover, \\ Germany
}

\begin{abstract}
This work describes a method to detect and to quantify any local or mid-range discontinuity on extended flat metal planes. Often these planes are used for antenna calibration (open area test site - OATS) or the plane could be the ground of a semi-anechoic chamber used in Electromagnetic Compatibility (EMC) testing. The measurement uncertainty of antenna calibration or EMC testing depends on the groundplane's quality, which can be accessed using this method.

A vector network analyzer with time-domain option is used to determine the complex-valued input scattering parameter $\underline{S}_{11, F}$ of an aperture antenna in a monostatic setup. $\underline{S}_{11, F}$ contains the information desired about the discontinuities and is measured in the frequency domain with high dynamic range. But only after a linear filtering utilizing the Chirp-Z-Transform the obtained time-domain signal $\underline{S}_{11, T}$ gives clear evidence of local and mid-range discontinuities.
\end{abstract}

\section{Introduction}

Calibrated antennas are used for EMC testing and in particular for emissions measurements. In the calibration process of antennas the antenna factor $A F$ is obtained, which converts the electrical field strength $E$ into a voltage $U$ across a $50 \Omega$ load resistor. For some applications the free-space $A F$ is needed, for others the height-dependent $A F$ is utilized. In theory the height-dependent $A F$ may be obtained best on an ideal large open area test site (OATS), which can be described as a lossless metal sheet, with infinite extension and with optical flatness in terms of a fraction of a wavelength. Real OATS are limited in size and the metal plane is constructed from single plates made from lossy material. The extended metal plane (groundplane) on a basement without or small elevation compared to the surrounding area may be assembled by different techniques. The individual parts can

Correspondence to: T. Schrader

(thorsten.schrader@ptb.de) either be screwed down tightly on a supporting structure underneath with a conducting foil filling the gap between the single plates or can be assembled by welding. Choosing the last, each welding has to be carried out very carefully during construction, otherwise steps in height crossing the edges of the plates will occur. Spot-wise welding will lead to a discontinuous metal plane, raised welding beads will behave as reflectors. All of the described defects can be subsumed as local discontinuities, adding their part to measurement uncertainty of the $A F$.

Another part of uncertainty is caused by the finite size of the groundplane. All fields emanating from a source on the OATS find boundary conditions determined by the groundplane itself. Suppose a very low-loss material, only vertical electrical fields and horizontal magnetic fields can exist in nearest vicinity of the metal plane. Surface currents and charges maintain the continuity of the fields at the interface of metal and the half space of air above. For the case of an ideal groundplane with dimensions extended to infinity the surface currents and charges on the metal plane are not distorted by a sudden change of the material's distribution. In such a case both the time-averaged and the instantaneous Poynting vector show only a uniform direction of propagation of electromagnetic waves generated on the OATS. At the edge or at the end of a real metallic groundplane the boundary conditions for electromagnetic waves are altered quite suddenly. This can be seen by a noticeable backward component of the Poynting vector. Therefore, special measures have to be taken to reduce the influence of this midrange discontinuity. The method described in this work can be used to obtain measurement data for the reflectivity of groundplane edges, which gives rise to measurement uncertainty again.

But local and mid-range discontinuities are not the only reasons for measurement uncertainty in calibration of antennas on large OATS stemming from the basic installation. We also have to look at the environment of such OATS, both in terms of the electromagnetics (interfering signals emanating from other sources) and the mechanics. For the moment we are only interested in the mechanical structure of the area 
encompassing the OATS. With increasing distance between the OATS and any trees, bushes, shelters, houses or other reflective items and installations like e.g. fences, these farrange discontinuities will contribute less and less to the uncertainty of measurement due to a very low signal level. But often the surrounding area can not be kept free of reflective items and the measurement setup should be able to detect far-range reflective parts even despite long delay time of the round-trip signals. Far-range discontinuities will be subject to another work and are not dealt with here.

Yet we have not discussed the influence of e.g. antenna towers, restraints, and cabling. Usually all materials being used as mechanical support in RF measurement show properties with complex dielectric constant. In order to carry bulky antennas the mechanics of supporting structures is designed as stiff as needed but as light as possible. Over the years glass reinforced plastics (GRP) and other materials with low dielectric constant have been developed to solve the problem of contradictory demands in RF mechanical setups. But still, influence of material's properties on the measurement data has to be taken into account, particularly in applications like antenna calibration. For the given purpose of antenna calibration on OATS, local, mid- and far-range discontinuities contribute to the measurement uncertainty. Discrepancies become especially visible, if measurement data is compared to theoretical numbers obtained by e.g. the Method of Moments or analytical solutions.

This work describes a measurement setup to detect local and mid-range discontinuities on OATS, their position and the order of influence. The method described can be used simultaneously with the construction progress of a groundplane as an inspection tool as well as for verification in a quality assurance program.

\section{Theory and Measurement Setup}

In order to detect the unwanted backward propagation of the Poynting vector the complex-valued input scattering parameter $\underline{S}_{11}$ of an antenna is measured as a function of frequency. Two different aperture antennas have been tested in the frequency range from $5.85 \mathrm{GHz}$ to $8.2 \mathrm{GHz}$. Among these are two commercially available antennas, a standard gain horn and a double ridged guide horn antenna. A $40 \mathrm{GHz}$ vector network analyzer (VNA) is utilized as instrumentation. Before any measurement the VNA is calibrated at the end of the coaxial cable in 1-port reflection. For all calculations later on, this plane is referred to as the reference plane, to which the phase of any signal is normalized. The two different types of antennas are connected consecutively to port 1 of the VNA, always with the plane of the aperture on a reference location. Each antenna is mounted close to the groundplane with the normal vector of its aperture parallel to the surface of the groundplane. The electrical field vector is always perpendicular to the groundplane, the magnetic field vector is parallel to it. Due to this arrangement we obtain a stacked antenna system by the antenna itself and the mirror object caused by the metallic groundplane. The radiated energy is effectively concentrated in the area of concern with the Poynting vector parallel and close to the surface of the groundplane. As the antenna is close to the metallic structure of the groundplane, the last determines the boundary conditions for the fields radiated in the half space above. In the ideal case a lossless, smooth and flat plane with infinite lateral dimensions guides the wave towards infinity without any reflection. Any disturbance of the ideal case generates a contribution to a reflected signal, which will add with respect to the phase and amplitude to the input scattering parameter $\underline{S}_{11, F}$ of the antenna in the frequency domain.

The measured frequency domain data is transformed to the time-domain using the Chirp-Z-transform (Ersoy, 1997) built into the VNA. A Hanning window is applied during the transformation. After normalization the time-domain data can be treated as a reflection in arbitrary units as function of distance to the reference plane. With time-domain gating the interesting band of distance can be selected and the magnitude of data vs. distance is stored afterwards for further calculations. For simplicity the data obtained is called $\underline{S}_{11, T}$ throughout the text.

Usually twice the distance between the reference plane and the antenna aperture is the start distance for the area which is looked at, counting from the reference plane. The unambiguous range in time-domain and, therefore, the stop distance is obtained from the VNA and depends on the frequency range applied. The Chirp-Z-transform provides an increased but finite range of valid values for the abscissa, which is a distance in this application. The advantage of this particular form of the Discrete Fourier Transform in the kind of linear filtering is the capability to choose time and frequency sampling intervals at will (Ersoy, 1997).

\section{Results}

In order to verify the obtained results of reflection vs. distance, simple but small objects have been positioned on the groundplane of a semi-anechoic chamber in a known distance to the antenna aperture. As test objects a) a thin metal plate of $0.7 \mathrm{~mm}$ thickness and $70 \mathrm{~cm} \times 28 \mathrm{~cm}$ length, b) a styrofoam plate of about $25 \mathrm{~cm}$ length and $2 \mathrm{~cm}$ thickness and c) a small $11 \mathrm{~mm}$ long screw have been used consecutively. By movement of one object at a time over a known distance, the change in $\underline{S}_{11, T}$ was recorded as well as $\underline{S}_{11, T, \text { empty }}$ without any of the aforementioned additional items. Moving one of the items towards the antenna's aperture or away from it, the curvature of $\underline{S}_{11, T}$ vs. distance will change at two spots. At the former place the line will drop to $\underline{S}_{11, T \text {,empty }}$ and at the new location the correspondent level will be increased to the level of reflection caused by the individual object (Fig. 1). The other spikes stem from other local discontinuities such as gaps between the single metal plates or access hatches for electricity and other connections needed in a semi-anechoic chamber. Note that in frequency domain the shift of $\underline{S}_{11, F}$ due to the present object is nearly not noticeable, while it can 


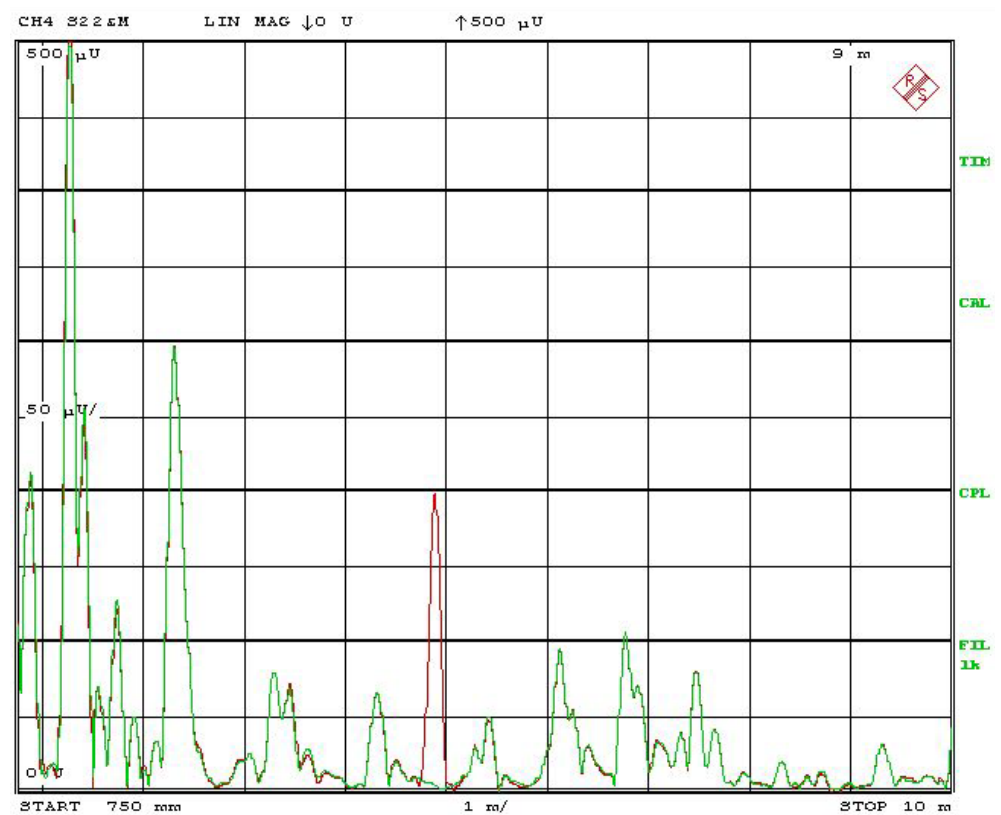

Fig. 1. Reflection in arbitrary units vs. distance measured with a standard gain horn antenna. The difference in both curves is caused by a test object (vertical screw on the ground, $11 \mathrm{~mm}$ height), giving rise to reflection in $4.86 \mathrm{~m}$ distance to the antenna's aperture (red spike). The green curve shows data for the same measurement setup, but without the test object. The other spikes stem from local discontinuities of the groundplane.

clearly be detected in the time domain response $S_{11, T}$. But still it is an advantage to utilize the dynamic range of frequency measurements, therefore, the source power level of the VNA of $10 \mathrm{dBm}$ is adequate. What is not included in the results yet is a correction for the site attenuation. Reflections from discontinuities far away from the antenna sustain a greater attenuation than those close to the antenna. The signals from the test object c) may be a first estimate for the attenuation vs. distance (Fig. 2), because the reflectivity of the objects remains the same for all positions, but the measured reflectivity level is decreasing. The attenuation follows $1 / r^{2}$.

In principle, the input scattering parameter $S_{11}$ of an antenna describes the superposition of internal reflections inside the antenna, reflections due to objects in front of the antenna weighted with the antenna pattern, and the influence of repeatability of coaxial connections. While the last can not be influenced by the setup except for using a precise PC- $7 \mathrm{~mm}$ connector, the influence of the antenna is investigated. Besides the internal reflections, also the antenna pattern, the height and the tilt angle of the plane of the antenna's aperture against the vertical direction may have an influence. To obtain the influence of different antennas, the same setup with test object c) (screw) was measured with each of the afore-mentioned antennas. All antennas are used with their aperture at the same location, but they differ in length of the flared section and their feeding waveguide, while the reference plane for the phase is maintained from calibration of the VNA. Therefore, the same object at the same position will be found in different distances from the

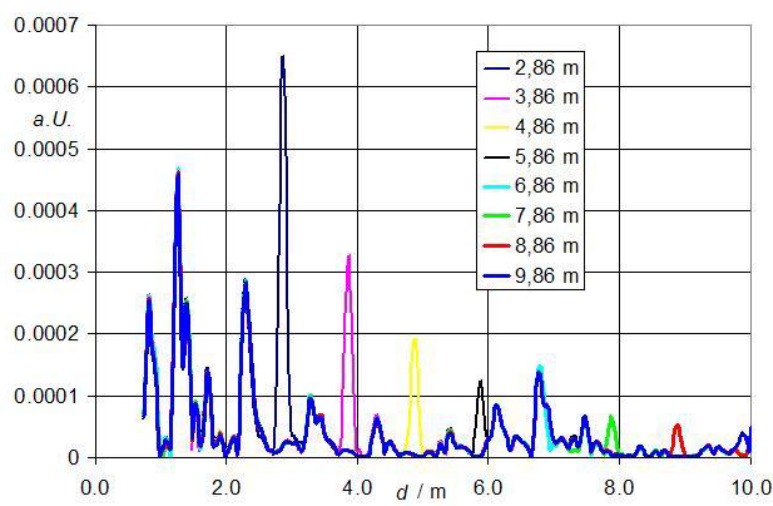

Fig. 2. Reflection (in arbitrary units) of a vertical screw with $11 \mathrm{~mm}$ length as a function of distance to the aperture of a standard gain horn antenna. The screw represents a local discontinuity. The decreasing level of reflection is due to site attenuation and is not corrected in this graph. It follows $1 / r^{2}$.

antenna's aperture, as can be seen in Fig. 3. What can also be seen is that reflections are much more pronounced using the standard gain horn. The reasons are a better defined radiation (and reception) pattern for a smaller solid angle and a closer match to the $50 \Omega$ of the feeding coaxial cable. The standard gain horn antenna turned out to be best suited for the given purpose, so we investigated tilt angle and height variations with it. For this purpose we compare the relative change on the different setups. 


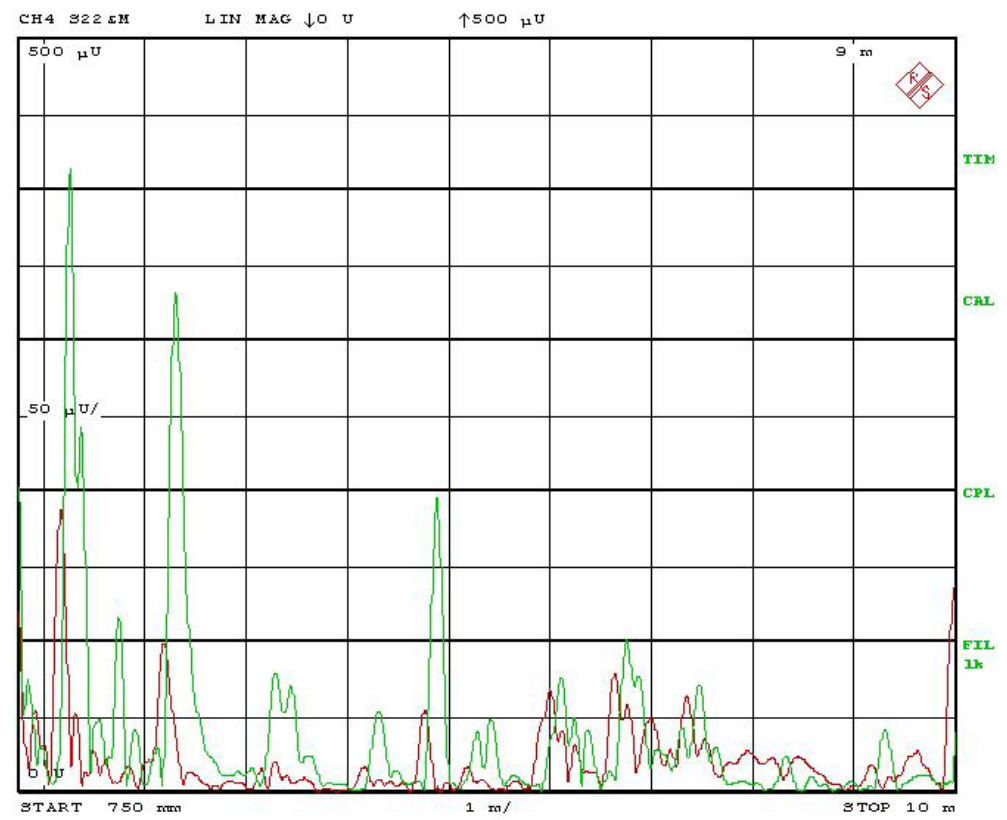

Fig. 3. Reflection in arbitrary units vs. distance measured with a standard gain horn antenna (green) and a double ridged guide horn antenna (red). The reflections are caused by local discontinuities of a groundplane in a semi-anechoic chamber used for emission measurements of Electromagnetic Compatibility. The shift vs. distance is due to different lengths of the antennas with respect to the same reference plane at the vector network analyzer port. The apertures of both antennas are at the same location.

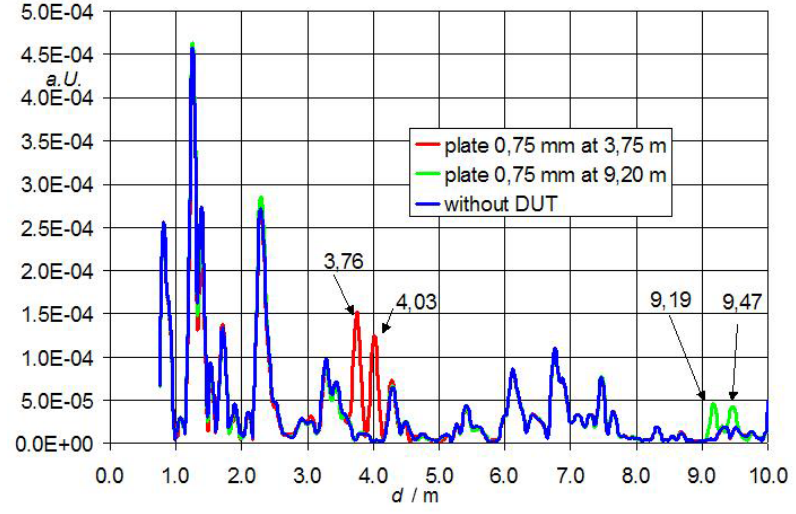

Fig. 4. Reflection in arbitrary units of a $0.75 \mathrm{~mm}$ metal plate on the metal groundplane. From the two setups with the plate $3.76 \mathrm{~m}$ and $9.19 \mathrm{~m}$ away from the antenna's aperture, the local resolution can be observed. Using frequency data in the range from $5.85 \mathrm{GHz}$ to $8.2 \mathrm{GHz}$, a resolution of $2 \mathrm{~cm}$ can be achieved, which is more than sufficient to localize any discontinuity of the groundplane.

Tilting angles of $\pm 5^{\circ}$ out of the vertical direction have been found already critical for a $10 \mathrm{~m}$ distance. Although for a $\mathrm{C}$ band standard gain horn antenna the half-power beam width (HPBW) in the E plane is around $25^{\circ}$ (Balanis, 1997), the spread of variation in $\underline{S}_{11, T}$ due to a varying tilt angle is equivalent to the reflection of test object c) in $10 \mathrm{~m}$ distance. We obtained the lowest reading for $+5^{\circ}$ and the highest for $-5^{\circ}$, while for $0^{\circ}$ the result was in between the others. The $\mathrm{HPBW}$ in the $\mathrm{H}$ plane is also about $25^{\circ}$. In $10 \mathrm{~m}$ distance from the horn aperture the beam has opened to a width of $2 \mathrm{~m}$. Any scattering object or local discontinuity can be localized on the radius within this solid angle. To localize it within the angular distance, a method of triangulation has to be applied. To access larger areas a scanning procedure has to be applied.

Varying now the height of the lower edge of the antenna's aperture to the ground, we obtained the highest reflection levels for $2 \mathrm{~cm}$ height and less for $0 \mathrm{~cm}, 5 \mathrm{~cm}, 13 \mathrm{~cm}$ and $23 \mathrm{~cm}$. We do not expect much more deviation for smaller distances. For measurement distances up to $10 \mathrm{~m}$ from the aperture a height of a few $\mathrm{cm}$ can be chosen arbitrarily and is not a critical parameter as long as a resistive contact to the groundplane is avoided and care is taken about the alignment of the antenna without boresight displacement.

The $0.7 \mathrm{~mm}$ thick metal plate lying flat on the ground as the second test object was spotted with its front and back edge at $3.76 \mathrm{~m}$ and at $9.19 \mathrm{~m}$ distance from the antenna's aperture (Fig. 4). From this data the local resolution can be obtained. Using frequency data in the range from $5.85 \mathrm{GHz}$ to $8.2 \mathrm{GHz}$, a resolution of $2 \mathrm{~cm}$ can be achieved, which is more than sufficient to localize any discontinuity of the groundplane.

One real test object is the installation of a turntable in a semi-anechoic chamber used for EMC emission measurements. Manufacturers of such devices design the junction between stationary and moving parts with sliding contacts to maintain a smooth transition for the RF surface currents. Applying the described procedure this property of turntables may be verified. Figure 5 gives an example of measurements 


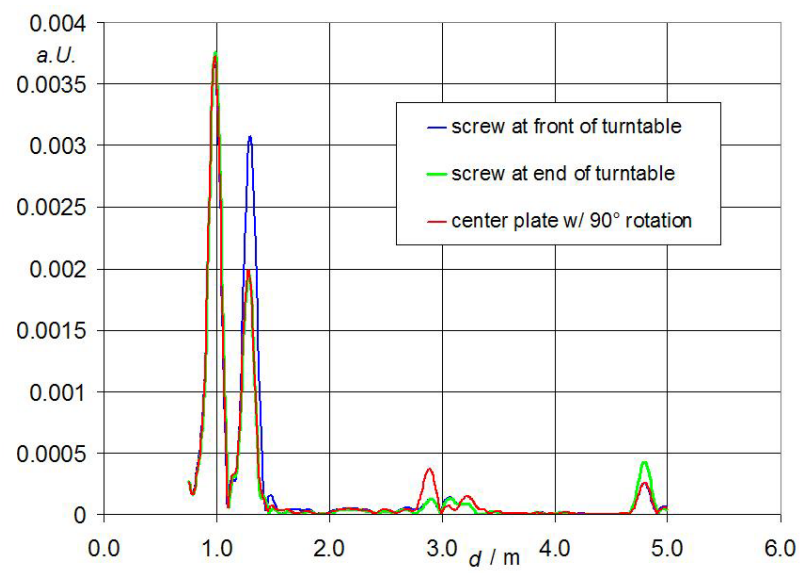

Fig. 5. Reflection in arbitrary units vs. distance of a turntable used in semi-anechoic chambers for EMC testing. To make any of the discontinuity more visible, the $11 \mathrm{~mm}$ long screw was set at different locations like begin and end of the turntable. The cover plate on the access hatch (center plate) can be closed in different orientations (here: rotation of about $90^{\circ}$ ), which can be noticed by different reflections of the grip holes.

on such a turntable. At first the transition from the groundplane to the rim of the turntable occurs as a spike, followed by the next transition from the rim to the turntable itself, to the moveable center plate covering electrical installation for test equipment and vice versa.

\section{Conclusion}

A method has been described to detect and to quantify any local or mid-range discontinuity on extended and flat metal planes utilizing a vector network analyzer with time-domain option. As a measure for the discontinuities the reflection level of a stacked antenna using an aperture antenna just above and its mirror image below the metal groundplane is used. The transmitted energy is focused by the mono-static setup and is travelling as a wave along the metal surface with the Poynting vector being parallel to it. The metal plane determines the boundary conditions for the wave which is continued by surface currents and charges on the metal surface. Any discontinuity disrupts the undistorted distribution of currents and charges and, therefore, the energy transport. The formerly unidirectional Poynting vector is superimposed with a backward component which is then received by the antenna. The complex-valued input scattering parameter $\underline{S}_{11, F}$ contains the superposition of the antenna's internal reflections and contributions from any object in front of the antenna and from the connector. It is obvious that well-matched standard gain horn antennas with their defined radiation and reception pattern fit best the given purpose. Although the frequency domain signal $\underline{S}_{11, F}$ contains all information we have been looking for, it can not be seen very easily. After a
Chirp-Z-transform the time-domain signal $\underline{S}_{11, T}$ gives clear evidence of local and mid-range discontinuities.

\section{References}

Balanis, C. A.: Antenna Theory, Second Edition, John Wiley and Sons, NY, ISBN 0-471-59268-4, 1997.

Ersoy, O.: Fourier-Related Transforms, Fast Algorithms and Applications, Prentice Hall, NJ, ISBN 0-13-624412-2, 1997. 